\title{
Comparison of three faecal occult blood tests in the detection of colorectal neoplasia
}

\author{
R L Hope, G Chu, A H Hope, R G Newcombe, P E Gillespie, S J Williams
}

\begin{abstract}
Methods and Aims-For the detection of colorectal neoplasia, 192 consecutive patients had colonoscopy to evaluate the sensitivity and specificity of three faecal occult blood tests (FOBT). Of 160 evaluable patients (96 female, mean age 51.9), 21 patients $(13 \cdot 1 \%)$ had adenomas and three patients $(1.9 \%)$ had colorectal carcinoma.

Results-When comparing all three faecal occult blood tests for the detection of colorectal neoplasia, the sensitivity of Monohaem (43.8\%) was superior to both Hemoccult II (25\%) and to BM-Test colon albumin $(25 \%)$. The specificity of Monohaem $(94.6 \%)$ was greater than both Hemoccult II (88\%) and BM-Test colon albumin (89\%). Using McNemar's test, Monohaem was a more accurate FOBT than Hemoccult II and BM-Test albumin $(p<0 \cdot 05)$. In the 21 patients with adenomatous polyps, FOBT sensitivity seemed to be dependent on polyp size, but not polyp site.

Conclusion-Monohaem, a feacal occult blood test that uses a monoclonal antibody that is specific for human haemoglobin, is a more accurate test in the detection of colorectal neoplasia and should possibly be used in colorectal cancer screening programmes.

(Gut 1996; 39: 722-725)
\end{abstract}

Keywords: colorectal neoplasia, faecal occult blood tests, Monohaem, Hemoccult II, adenoma, screening.

Screening by means of faecal occult blood testing (FOBT) has been proposed to reduce the incidence of and mortality from colorectal cancer. Tests include the traditional guaiac test, which detects the pseudoperoxidase activity of heme, and immunochemical tests specific for human haemoglobin and human albumin.

The guaiac test, Hemoccult II (HOII) (Smith Kline Diagnostics, San Jose, CA) is the most common test used in colorectal screening programmes. ${ }^{1-4}$ It, however, has a low level of sensitivity for asymptomatic colorectal cancer. ${ }^{12}{ }^{5}$ For colorectal adenomas, the sensitivity is even lower, probably because of a fluctuation in, or absence of, occult blood loss.

Repeated testing using HOII does increase the sensitivity of the screening procedure. However, recent attention has been directed toward newer immunochemical tests, which detect haemoglobin at lower concentrations. ${ }^{6-9}$ Monohaem (MH) (Silenus Lab Pty Ltd, ICI
Australia Ltd) uses a monoclonal antibody that is specific for human haemoglobin, which theoretically should decrease the number of false positive test results. The BM Test colon albumin (BMCA) (Boehringer Mannheim, UK) is designed to detect human albumin present in the faecal sample, which may be associated with colorectal neoplasia.

The aim of this study is to evaluate the sensitivity and specificity of these three FOBTs as a means of detecting colorectal neoplasia.

\section{Methods}

\section{Patients}

From March 1991 until November 1992, 192 consecutive patients who required colonoscopy for the investigation of gastrointestinal symptoms or for 'polyp surveillance' follow up (see Figure), were recruited for study. In no case was colonoscopy being performed for previously positive FOBTs and patients with a history of overt gastrointestinal bleeding were excluded. No patient had undergone previous colonic resectional surgery. Patients were asked to start the testing of stools five days before hospital admission to ensure enough time to complete the three day regimen before bowel preparation. There were no dietary restrictions placed on the patients and regular medications were continued.

\section{Study design}

The initial 56 patients were asked to prepare two FOBT kits: (a) Monohaem screen included one card per day over three days and (b) Hemoccult II screen, which included one card per day over three days. The ensuing 136

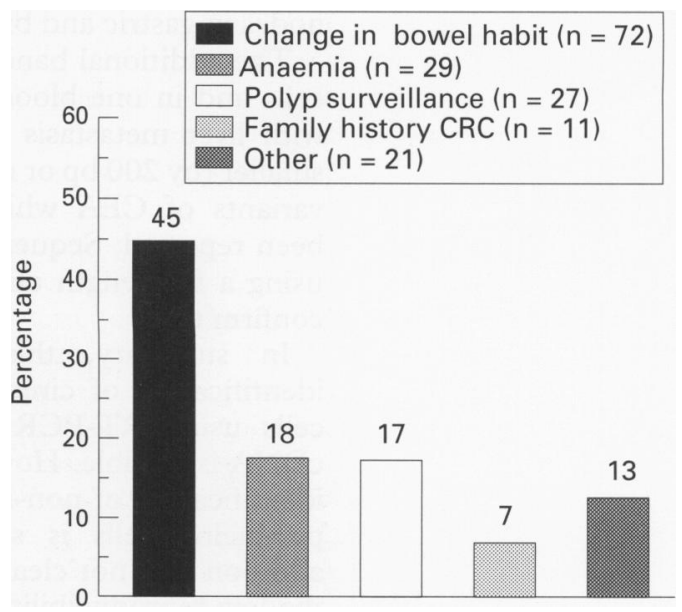

Major indication for colonoscopy in 160 patients.

Department of
Gastroenterology,
Westmead Hospital,
Westmead, Australia
R L Hope
G Chu
A H Hope
P E Gillespie
S J Williams
Department of Medical
Computing and
Statistics, University
of Wales College of
Medicine, Cardiff,
Wales
R G Newcombe
Correspondence to:
Dr R L Hope,
Department of
Gastroenterology, Westmead
Hospital, Sydney, NSW,
2145 Australia.
Accepted for publication
20 June 1996


patients were asked to prepare three FOBT kits: (a) Monohaem Screen (as above), (b) Hemoccult screen (as above), and (c) BM-Test colon albumin screen, which included two cards each day over three days.

Monohaem uses mouse monoclonal antibodies covalently linked to the Monohaem strip that specifically bind human haemoglobin to detect abnormal amounts of occult blood in stool samples. After application of the sample to the card, the bound haemoglobin is detected by treatment with an aqueous ethanolic solution of gum guaiac followed by hydrogen peroxide. The gum guaiac resin contains $\alpha$ guaiaconic acid, which is oxidised in the presence of peroxide to a blue coloured product by the pseudoperoxidase enzymatic action of haemoglobin. Because the haemoglobin is bound selectively by the localised monoclonal antibody on the card, a positive reaction appears as a blue ring or spot confined to the area of antibody immobilisation. ${ }^{10}$ The assessment of the colour change is done visually, five to 60 seconds after the application of the hydrogen peroxide solution and there is little ambiguity in the interpretation of a positive or negative test. The antibody specifically selects for human haemoglobin, thus eliminating false positives from other peroxidase active agents.

The Hemoccult II kit consists of a peroxidase substrate impregnated card, which is developed with hydrogen peroxide after application of a faecal smear. Any haemoglobin present in the faecal smear gives a blue coloured reaction by virtue of its pseudoperoxidase activity. The coloured reaction is evident anywhere on the card where haemoglobin is present. As there is no specificity for human haemoglobin, any other peroxidase present can give rise to a positive reaction. HOII test was performed unhydrated.

The BM-Test colon albumin kit consists of a card that is probed with a strip carrying sequentially arranged reagent fields designed to detect any human albumin present in the faecal sample. Elution from the sample proceeds to formation of a complex with galactosidase conjugated antihuman albumin monoclonal antibody and then on to a dye conjugated galactoside releasing the dye to give a colorimetric end point if human albumin is present. A red to violet colouration constitutes a positive result, and yellow to orange-brown colouration constitutes a negative result. The colour change is assessed visually, five to 15 minutes after the reagent is added.

All participants were given verbal and written instructions in the method of specimen collection. Stools were collected on several layers of toilet paper on a folded newspaper, disposable container or child's potty, ensuring that the stool was not contaminated with water. Samples for the tests were collected immediately afer defecation and using a spatula, the windows on the cards were well covered with faecal smear (a drop of water was added to the window on the Monohaem card). A different section of the same stool was sampled to inoculate a second window on the Hemoccult and Monohaem cards. After sampling, the cards were sealed, labelled, and stored in a cool dry place. Patients returned the completed FOBT kits on hospital admission for the colonoscopy.

Kits were analysed at an independent laboratory (Sugerman's Pathology Pty Ltd, Hurstville, NSW, Australia) to ensure that the colonoscopist was not aware of the FOBT result. Analysis was performed within 48 hours of arrival at the laboratory and, therefore, all kits were processed within 10 days of stool sampling. All kits were processed according to manufacturers' instructions. A single positive smear was considered a positive test.

Colonoscopy was performed (by SJW, PEG or under their direct supervision) without knowledge of the FOBT result. If colorectal polyps were detected, polyp site was recorded and polypectomy performed. Polyps were examined histologically and size recorded. Colorectal cancer site and histology was recorded when found.

\section{Results}

Overall, 160 patients $(83.3 \%)$ were evaluated as they had completed FOBT kits correctly and had total colonoscopy (to the base of the caecum). Twenty six patients $(13.5 \%)$ had inoculated the FOBTs incorrectly and were not included in the analysis. Six patients $(3 \cdot 1 \%)$ were excluded from analysis as they had either very poor bowel preparation (three patients) or the colonoscopy was incomplete (three patients).

There were 96 women and 64 men, with a mean age of 51.9 (range 24-89). The Figure shows the indication for colonoscopy. Fifteen patients had hyperplastic polyps $(9 \cdot 4 \%), 21$ patients $(13 \cdot 1 \%)$ had adenomas (11 proximal to splenic flexure, 10 distal to splenic), and colorectal carcinoma occurred in three patients ( $1.3 \%$ rectosigmoid, $0.6 \%$ hepatic flexure).

\section{Test sensitivity for colorectal carcinoma}

Overall, three patients had colorectal carcinoma. Both $\mathrm{MH}$ and HOII were positive in these three patients, however, BMCA was negative in one patient with a rectosigmoid carcinoma.

\section{Test sensitivity for adenoma}

Overall, 21 patients had adenomas. The sensitivity of $\mathrm{MH}(52.4 \%)$ was greater than HOII $(28 \cdot 5 \%)$. If a patient had more than one adenoma (12 patients), the most distal polyp site was recorded. The sensitivity for detecting adenomas (using $\mathrm{MH}$ and HOII) did not seem to be dependent on polyp site (see Table I), although the absolute number of patients with adenomas was small.

The mean adenoma size was $11 \mathrm{~mm}$ (range 3-30). The sensitivity of $\mathrm{MH}$ was greater in patients with large $(\geq 10 \mathrm{~mm})$ adenomas $(70 \%)$ than in patients with small $(<10 \mathrm{~mm})$ adenomas $(36.4 \%)$. The same trend in FOBT sensitivity was seen with regard to polyp size using HOII 
TABLE I Influence of adenoma site and size on FOBT

\begin{tabular}{llll}
\hline Adenoma & & $\begin{array}{l}\text { Monohaem } \\
\text { sensitivity } \\
(\%)\end{array}$ & $\begin{array}{l}\text { HOII } \\
\text { sensitivity } \\
(\%)\end{array}$ \\
\hline $\begin{array}{l}\text { Site (relative to } \\
\text { splenic flexure) }\end{array}$ & $\begin{array}{l}\text { Proximal } \\
(\mathrm{n}=11)\end{array}$ & $54 \cdot 5$ & $27 \cdot 3$ \\
Size & $\begin{array}{l}\text { Distal } \\
(\mathrm{n}=10)\end{array}$ & 50 & 30 \\
& $\begin{array}{l}<10 \mathrm{~mm} \\
(\mathrm{n}=11) \\
\geqslant 10 \mathrm{~mm} \\
(\mathrm{n}=10)\end{array}$ & $36 \cdot 4$ & $9 \cdot 1$ \\
\hline
\end{tabular}

(sensitivity $36 \cdot 4 \%$ for large adenomas and $9 \cdot 1 \%$ for small adenomas (see Table I)).

Hemoccult II versus Monohaem comparison

A total of 160 patients were evaluated to compare HOII versus Monohaem (Tables II and III). For detecting colorectal neoplasia (that is, adenomatous polyps or colorectal carcinoma), the sensitivity of $\mathrm{MH}(58.3 \%)$ was superior to HOII $(37 \cdot 5 \%)$ with $95 \%$ confidence intervals for difference in sensitivity -0.075 to +0.451 (unpublished, submitted data). The specificity of $\mathrm{MH}(96 \cdot 3 \%)$ was also superior to HOII $(86.8 \%)$ with $95 \%$ confidence intervals for difference in specificity +0.037 to $+0 \cdot 162$. Consequently, positive and negative predictive values of $\mathrm{MH}(73.7 \%$ and $92.9 \%$ in this series) were superior to those of HOII (33.3\% and $88 \cdot 7 \%)$.

\section{Monohaem versus BM-Test colon albumin comparison}

Some 108 patients were evaluated to compare $\mathrm{MH}$ versus BMCA (Tables IV and V). For

TABLE II McNemar table for 24 affected patients (with colorectal neoplasia) comparing $M H$ versus $H O I$

\begin{tabular}{llcl}
\hline & HOII+ & HOII- & \\
\hline MH+ & 5 & 9 & 14 \\
MH- & 4 & 6 & 10 \\
& 9 & 15 & 24 \\
\hline
\end{tabular}

TABLE III McNemar table for 136 unaffected patients (no colorectal neoplasia) comparing $M H$ versus $H O I I$

\begin{tabular}{lccr}
\hline \multicolumn{3}{c}{ HOIt } & HOII- \\
\hline MH+ & 3 & 2 & 5 \\
MH- & 15 & 116 & 131 \\
& 18 & 118 & 136 \\
\hline
\end{tabular}

TABLE IV McNemar table for 116 affected patients (with colorectal neoplasia) comparing $M H$ versus $B M C A$

\begin{tabular}{llcr}
\hline & $B M C A+$ & $B M C A-$ \\
\hline MH+ & 3 & 4 & 7 \\
MH- & 1 & 8 & 9 \\
& 4 & 12 & 16 \\
\hline
\end{tabular}

TABLE V McNemar table for 92 unaffected patients (no colorectal neoplasia) comparing $M H$ versus $B M C A$

\begin{tabular}{lccr}
\hline \multicolumn{3}{c}{$B M C A+$} & $B M C A-$ \\
\hline MH+ & 2 & 3 & 5 \\
MH- & 8 & 79 & 87 \\
& 10 & 82 & 92 \\
\hline
\end{tabular}

detecting colorectal neoplasia, the sensitivity of $\mathrm{MH}(43 \cdot 8 \%)$ was greater than BMCA (25\%) with $95 \%$ confidence intervals for difference in sensitivity -0.096 to +0.433 . The specificity of $\mathrm{MH}(94 \cdot 6 \%)$ was also greater than BMCA $(89 \cdot 1 \%)$ with $95 \%$ confidence intervals for difference in specificity $-0 \cdot 022$ to $+0 \cdot 136$.

\section{Discussion}

The ideal screening test on a population should combine satisfactory sensitivity with high specificity. Unfortunately, Hemoccult II has quite poor sensitivity for detecting asymptomatic colorectal neoplasms. ${ }^{211}$ While rehydration of the faecal sample does increase the sensitivity of HOII, it does so at the expense of decreasing specificity. ${ }^{5}$ This loss in test specificity can generate significant burden on a colorectal screening programme and therefore, rehydration is not routinely performed. ${ }^{12-14}$

Our study has shown that the immunochemical test, Monohaem, is both more sensitive and more specific for detecting colorectal neoplasms than Hemoccult II. It is a more accurate FOBT. This is important, as minor changes in test sensitivity and specificity can have an important impact on efficiency and cost effectiveness of a screening programme. ${ }^{6}$

St John et $a l^{15}$ suggest that the better performance of HemeSelect (another immunochemical test) related to its high chemical sensitivity for haemoglobin (in vitro) and the specificity of the test for human haemoglobin. Immunoreactive haemoglobin is rapidly degraded in the stomach and duodenum, ${ }^{16}$ giving these tests a high degree of selectivity for bleeding from the distal gastrointestinal tract. The other obvious advantage of an immunochemical test directed against human haemoglobin is that false positive results do not occur when peroxidase containing materials have been ingested. Red meat and fruit and vegetables containing peroxidase need to be avoided when Hemoccult $\mathrm{II}^{1718}$ is used, which may diminish patient compliance. ${ }^{19}$ It is not necessary to restrict the diet before Monohaem testing.

Macrae and St John ${ }^{18}$ showed that only large adenomas with a diameter of $>2 \mathrm{~cm}$ cause faecal blood loss of $>2 \mathrm{ml} /$ day. The superior sensitivity of both HOII and $\mathrm{MH}$ for detecting larger polyps was in keeping with this finding. Given that most colorectal cancers arise from preexisting adenomas (adenoma-adenocarcinoma sequence), ${ }^{2021}$ the detection of large adenomas (and subsequent removal) in an asymptomatic population is an important aim. ${ }^{22}$

Our patients were recruited for study after the decision to perform colonoscopy had been made. While our study was not on an asymptomatic population, we were able to obtain valuable information with regard to FOBT specificity. This has been a criticism ${ }^{23}$ of previous investigators using symptomatic subjects with known colorectal neoplasms. Specificity, defined as the proportion of screened people without pre-clinical disease who are designated correctly as negative, ${ }^{24}$ was 
able to be compared between the three FOBTs. Monohaem specificity $(94.6 \%)$ was superior to both HOII (88\%) and BMCA $(89.1 \%)$ in 92 patients who were truly free of colorectal neoplasia. These data on FOBT specificity are useful when we consider that the mean age of these patients was $52 \cdot 3$. While recommendations for FOBT screening on an asymptomatic population remain to be established, most would agree that screening will target an 'average risk' asymptomatic person between the age of 50-75.

Many factors including accuracy, cost effectiveness, ${ }^{25}$ and patient and physician compliance influence the selection of a faecal occult blood test for screening programmes. While cost effectiveness was not a primary aim of our comparative study, it is worth noting that the Monohaem screen is comparatively inexpensive. The initial outlay of approximately US $\$ 2$ (including card and reagent) per $\mathrm{MH}$ test, should be recouped by being a more specific test, and therefore not generating as many costly second phase tests such as colonoscopy.

In summary, the immunochemical test, Monohaem, was the most accurate faecal occult blood test. It combines high test sensitivity for detecting colorectal neoplasia with high test specificity. It should be considered as a more accurate FOBT and possibly used in colorectal cancer screening programmes.

1 Hardcastle JD, Thomas WM Chamberlain J, Pye G, Sheffield J, James PD, et al. Randomised controlled trial of faecal occult blood screening for colorectal cancer; results of first 107,349 subjects. Lancet 1989; i: 1160-4.

2 Allison JE, Feldman R, Tekawa IS. Hemoccult screening in detecting colorectal neoplasm: sensitivity, specificity and predictive value. Long-term follow-up in a large group practice setting. Ann Intern Med 1990; 112: 328-33.

3 Flehinger BJ, Herver E, Winawer SJ, et al. Screening for colorectal cancer with faecal occult blood test and sigmoidoscopy: preliminary report of the colon project of Memorial Sloan-Kettering Cancer Centre and PMIStrang Clinic. In: Chamberlain J, Miller AB, eds. Screening for gastrointestinal cancer. Toronto: Hans Huber, 1988: 9-16.

4 Winawer SJ, Schottenfeld D, Flehinger BJ. Colorectal cancer screening. F Natl Cancer Inst 1991; 83: 243-53.

5 Kronberg O, Fenger C, Olsen J, Bech K, Søndergaard O. Repeated screening for colorectal cancer with faecal occult blood test: a prospective randomised study at
Funen, Denmark. Scand $\mathcal{f}$ Gastroenterol 1989; 24: 599-606.

6 Walker AR, Whynes DK, Hardcastle JD. Rehydration of guaiac-based faecal occult blood tests in mass screening for colorectal cancer: an economic perspective. Scand $\mathcal{F}$ Gastroenterol 1991; 26: 215-8.

7 Yoshinaga M, Motomura S, Takeda H, YanagisawaZ, Ikeda K Evaluation of the sensitivity of an immunochemical fecal occult blood test for colorectal neoplasia. Am $\mathcal{f}$ Gastroenterol 1995; 90: 1076-9.

8 Robinson MHE, Kronborg O, Williams CB, Bostock K, Rooney PS, Hunt LM, et al. Faecal occult blood testing and colonoscopy in the surveillance of subjects at high risk of colorectal neoplasia. Br $\mathcal{\text { S Surg 1995; 82: 318-20. }}$

9 Robinson MHE, Marks CG, Farrands PA, Thomas WM Hardcastle JD. Population screening for colorecta cancer: comparison between guaiac and immunological

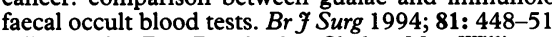

10 Marlborough D, Pucci A, Slade M, Williams M Development of a test for occult human haemoglobin using a monoclonal antibody. Proceedings of Australian Biochemistry Society 1984; 17: 46.

11 Griffith CDM, Jurner DJ, Saunders JH. False-negative results of Hemoccult test in colorectal cancer. BMF 1981; 283: 472 .

12 Winawer SJ, Flehinger BJ, Schottenfeld D, Miller DG Screening for colorectal cancer with fecal occult blood testing and sigmoidoscopy. $\mathcal{F}$ Natl Cancer Inst 1993; 85: 1311-8.

13 Selby JV, Friedman GD, Quesenberry CP, Weiss NS. Effect of fecal occult blood testing on mortality from colorecta cancer. Ann Intern Med 1993; 118: 1-6.

14 Wahrendorf J, Robra BP, Wiebelt $\mathrm{H}$, et al. Effectiveness of colorectal cancer screening results from a populationbased case-control evaluation in Saarland, Germany. Eur $\mathfrak{f}$ Cancer Prev 1993; 2: 221-7.

15 St John DJB, Young GP, Alexeyeff MA, Deacon MC, Cuthbertson AM, Macrae FA, et al. Evaluation of new occult blood tests for detection of colorectal neoplasia. Gastroenterology 1993; 104: 1661-8.

16 Burton RM, Landreth KS, Barrows GH, Jarrett DD, Songster CL. Appearance, properties, and origin of altered hemoglobin in feces. Lab Invest 1976; 35: 111-5.

17 Bassett ML, Goulston KJ. False positive and negative Hemoccult reactions on a normal diet and effect of diet restriction. Aust NZ F Med 1980; 10: 1-4

18 Macrae FA, St John DJB, Calagiore P, Taylor LS, Legge WJ Optimum dietary conditions for Hemoccult testing. Gastroenterology 1982; 82: 899-903.

19 Robinson MHE, Pye G, Thomas WM, Hardcastle JD, Mangham CM. Haemoccult screening for colorectal cancer: the effect of dietary restriction on compliance. Eur f Surg Oncol 1994; 20: 545-8.

20 Atkin WS, Morson BC, Cuzick J. Long term risk of colorectal cancer after excision of rectosigmoid colorectal cancer after excision of rect

21 Stryker SJ, Wolff BG, Culp CE, et al. Natural history of untreated colonic polyps. Gastroenterology 1987; 93 1009-13.

22 Winawer SJ, Zauber AG, Ho MN, et al. Prevention of colorectal cancer by colonoscopic polypectomy. $N$ Engl f Med 1993; 119: 836-43.

23 Lance P. Fecal occult blood tests: what's new? Gastroenterology 1993; 104: 1852-5.

24 Morrison AS. Screening in chronic disease. New York: Oxford University, 1985.

25 Robinson MHE, Marks CG, Farrands PA, Whynes DK Bostock K, Hardcastle JD. Is an immunological faecal occult blood test better than haemoccult? A cost-benefit study. Eur ₹ Surg Oncol 1995; 21: 261-4. 